072

\section{TOXOPLASMOSI OCULARE IN LINFOMA}

Grandi G. ${ }^{2}$; Bay A. '; Albanesi T.'; Melchio P.'; Trasendi P.';

'Divisione Glaucomi - Ospedale Oftalmico, Via Juvarra 19, 10100 Torino.

${ }^{2}$ Servizio di Patologia Clinica - Ospedale Oftalmico, Via Juvarra 19, 10100 Torino.

Paziente giunta in P.S. oculistico dell'Ospedale Oftalmico di Torino per alterazioni del visus. Viene posta diagnosi di vitreite dell'occhio sx. L'anamnesi patologica segnala presenza di linfoma non Hodgkin diffuso B di grandi cellule stadio IA diagnosticato da circa un anno. La paziente è stata sottoposta a chemioterapia R . CHOP. All'ecografia oculare si riscontrano disomogeneità vitreali dell'occhio sx.

L'esame fluorangiografico segnala un focolaio di corioretinite attivo fortemente suggestivo di lesione toxoplasmica. Gli esami sierologici per la toxoplasmosi danno i seguenti risultati: IgG negative (Bio Merieux Vidas), IgM 1,33 (positive $>0,65$ ) (Bio Merieux Vidas), Ig Totali in immunofluorescenza indiretta $<10 \mathrm{UI} / \mathrm{ml}$ (negative). Per confermare la specificità delle $\operatorname{IgM}$ in Elfa viene eseguito il test IgM ISAGA $=6$ (positività dubbia).

Possibili interpretazioni:

1. quadro sierologico di toxoplasmosi atipico a causa della neoplasia

2. quadro sierologico non significativo.

Il centro di parassitologia dell'Hopital de la Croix Rousse di Lione, col quale collaboriamo da anni, ci segnala come più probabile un quadro di toxoplasmosi atipico.

Sulla base del quadro fluorangiografico fortemente suggestivo di lesione da toxoplasma la paziente viene trattata con Trimetoprim sulfametossazolo e con Betametasone fosfato disodico. Si decide di ricontrollare il quadro sierologico dopo un mese. Questi i rusultati: IgG negative, IgM 1,71, IgM ISAGA 12 (positività massima), Ig Totali in immunofluorescenza indiretta dubbie / debole positività. La netta positivizzazione delle IgM ISAGA e una debole positività in IFI, associate ad un miglioramento in seguito a terapia confermano la diagnosi di focolaio di corioretinite toxoplasmico con sierologia atipica causata dalla neoplasia di base. Un pannello di esami sierologici di I e II livello, un dialogo costruttivo fra colleghi di branche diverse e la collaborazione con altri centri di riferimento europei permette una diagnosi corretta.

\section{3}

\section{LEISHMANIOSI VISCERALE IN PAZIENTE IMMUNOCOMPETENTE: CASO CLINICO}

Nisticò S.', Leone R.A.', Minchella P.', Potente G.I.', Borelli A.', Caruso V.', Caruso D.', Camerino M.', Carlei M.I.', Folino C.', Piccoli M.', Nicolazzo A.', Berardelli G. ${ }^{2}$, Petronio A. ${ }^{2}$, Luciano A.'

'U.O. Microbiologia e Virologia,

2U.O. Malattie Infettive,

Azienda Sanitaria N. 6, Via Perugini, 88046 Lamezia Terme (CZ)

Introduzione. Le leishmaniosi, infezioni causate da protozoi parassiti del genere Leishmania, possono clinicamente manifestarsi sotto tre forme: leishmaniosi cutanea, mucocutanea e viscerale. Le prime due possono essere considerate manifestazioni a decorso benigno, mentre la forma viscerale ha manifestazioni sistemiche gravi e si presenta generalmente nei soggetti immunocompromessi. Le forme flagellate del protozoo (promastigoti) vengono trasmesse all'uomo tramite puntura di artropodi vettori e si moltiplicano nei macrofagi come forme aflagellate (amastigoti). In Italia la leishmaniosi è endemica in tutte le regioni tirreniche, nel basso Adriatico e nelle isole. Il ceppo più comunemente presente in queste aree è rappresentato da Leishmania infantum. Scopo del lavoro è descrivere il caso di un paziente di sesso maschile di 41 anni, presentatosi all'osservazione dell'U.O. di Malattie Infettive, che da circa quattro mesi lamentava una sintomatologia caratterizzata da calo ponderale (circa $20 \mathrm{~kg}$ ), febbricola serotina, astenia e sudorazione notturna. Gli esami ematochimici, prontamente eseguiti in prima giornata, mostravano una marcata anemia ( $\mathrm{Hb} 11 \mathrm{mg} / \mathrm{dl}$ ), lievi disordini della coagulazione, aumento della VES, leucopenia (27,8\% neutrofili, 59\% linfociti), fosfatasi alcalina $307 \mathrm{UI} / 1$, GGT 216 UI/1, AST 54UI/1, ALT 61UI/1; l'elettroforesi delle proteine mostrava una diminuzione dell'albumina $(34,7 \%)$ ed un aumento della frazione gamma $(47,9 \%)$, con un rapporto alfa/gamma di 0,53. L'esame ecografico dell'addome evidenziava un'epatosplenomegalia. Posto il sospetto di infezione protozoaria da Leishmania si procedeva a prelievo sierologico per la ricerca di anticorpi anti-leishmania IgG ed anti-HIV $1 / 2$, nonché aspirato midollare osseo.

Metodi. A) Leishmania infantum IgG EIA, (Ditta Novatec, distribuito da RADIM); B) Axsym HIV $1 / 2$ gO, metodo Meia (Ditta Abbott); C) colorazione dello striscio di aspirato midollare (metodo Giemsa).

Risultati. L'esame istologico dimostrava la presenza di numerosi elementi intraistiocitari ed extracellulari morfologicamente riconducibili ad amastigoti di Leishmania. L'esame sierologico per la ricerca di anticorpi antiLeishmania di classe IgG dava esito positivo, mentre la ricerca di anticorpi anti-HIV $1 / 2$ ed Ag p24 dava esito negativo. Conclusioni. Il caso descritto dimostra che, pur essendo la leishmaniosi viscerale poco frequente negli immunocompetenti, tale infezione parassitaria è da tenere sempre in considerazione per evitare quei ritardi diagnostici che potrebbero rivelarsi fatali. 